\title{
Does enucleation of the eye containing a malignant melanoma prevent or accelerate the dissemination of tumour cells?
}

\author{
LORENZ E. ZIMMERMAN, IAN W. MCLEAN, AND WALTER D. FOSTER \\ From the Armed Forces Institute of Pathology, Washington, USA
}

SUMMARY A reappraisal of survival data on patients with uveal melanomas has led us to these impressions: $(a)$ that the mortality rate before enucleation is low, estimated at $1 \%$ per year; $(b)$ that the mortality rate rises abruptly following enucleation, reaching a peak of about $8 \%$ during the second year after enucleation; and $(c)$ that approximately two-thirds of the fatalities could be attributed to the dissemination of tumour emboli at the time of enucleation. From these impressions we believe the following conclusions are warranted: $(a)$ enucleation as it has been performed in the past may have for many patients an adverse rather than a beneficial effect with respect to the development of metastatic disease from malignant melanoma of the choroid and ciliary body. (b) A long-term follow-up study of untreated patients with melanomas of the choroid and ciliary body is indicated. (c) New techniques for enucleation designed to prevent the dissemination of tumour cells must be developed and tested to enable the ophthalmic surgeon to remove safely the tumour-containing eye that has developed such complications as uncontrollable glaucoma, panophthalmitis, or proptosis from extraocular extension.

Recently in reviewing lantern slides while preparing for a lecture on uveal melanomas, the senior author observed for the first time that the old, previously published graph shown in Fig. 1 (Paul et al., 1962; Zimmerman, 1967), illustrating the prognostic significance of cell type, seemed to contain hidden information that had heretofore escaped recognition. We interpreted and spoke as if time zero were the beginning of an experiment. If these curves represented survival of animals after the introduction of different types of melanoma cells at time zero, then all would be clear. What has often failed to be recognised in evaluating such survival curves is that there is no way of knowing when the biological experiment began, how many additional cases were diagnosed but not treated by enucleation, or how many undiagnosed cases there may have teen, including those that may have proved fatal. As a matter of fact it would seem quite certain that the time of onset is an extremely variable factor, often antedating by years the time of discovery and enucleation. To appreciate the hidden significance

Address for reprints: Dr Lorenz E. Zimmerman, The Armed Forces Institute of Pathology, Washington, DC 20306, USA of the data presented in Fig. 1 fully, one must consider the following background information.

First, it is generally believed and widely taught that uveal melanomas, unlike cutaneous melanomas and unlike many other malignant tumours, are rarely observed to have metastasised before the patient is referred for treatment of the primary tumour (Hogan, 1964; Duke-Elder and Perkins, 1966). The pre-enucleation clinical and radiological evaluations also seldom turn up any evidence of metastatic disease (Jensen, 1963), and necropsy studies rarely reveal uveal melanomas that had escaped clinical discovery. In a review of 1146 cases of uveal melanoma on file in the Registry of Ophthalmic Pathology, received from 1950 to 1960 , we found only 4 in which the patient had died with metastatic melanoma and had not had an enucleation. In these 4 cases the tumour-containing eye was obtained post mortem. In the remaining 1142 surgically enucleated eyes the tumours varied greatly in size, in the extent of damage they had produced to intraocular structures, and in their degrees of trans-scleral invasion. Some tumours caused no symptoms, having been discovered in the course of a routine examination, while others were far advanced with 


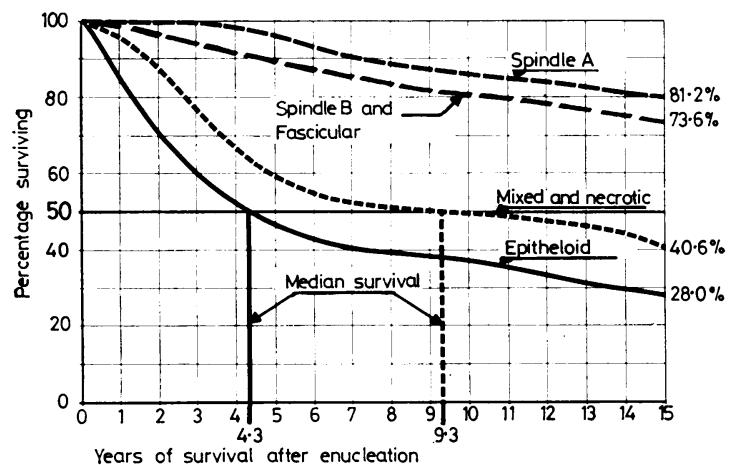

Fig. 1 Actuarial curves published by Paul et al. (1962) graphically illustrating the relationship of survival to cell type in a series of 2652 patients who had been treated by enucleation of the melanoma-containing eye. Observe the very steep downward slope of the curves for melanomas of epithelioid or mixed cell types beginning soon after enucleation. AFIP Neg. 61-6122

extraocular extension. It would be most illogical to assume that these tumours had all been growing for the same period of time before the surgeon interrupted their natural course by enucleating the eye. On the contrary, it would seem almost certain that the larger tumours had been growing for a much longer period than the smaller tumours. If there were a firm basis for the prevalent belief that it is important to treat a turmour-containing eye as soon as possible after the tumour is discovered, and if there were a markedly increased mortality rate associated with greater duration, one should expect to find amoung patients discovered to have very large tumours that there would be a significant number with metastatic disease. That, however, has not been our experience, nor has it been the experience of others. Regardless of how large a tumour has grown and whether or not extraocular extension may have occurred before the patient is first examined by an ophthalmologist, it is very unusual for clinical evaluation to reveal evidence of metastatic disease.

Another reason for believing there is not a high mortality rate among patients with untreated uveal melanomas is the experience of those who have had an opportunity to make extended observations of untreated tumours (Koenig, 1954; Anderson and O'Neill, 1957; Char and Hogan, 1977; Curtin, 1977; Dunphy, 1977; Gass, 1977; Hagler et al., 1977). Obviously such cases constitute a small percentage of the total because it has so long been clinical dogma that one must enucleate the tumour-containing eye as soon as possible. Nevertheless, because some patients have refused treatment, or because the other eye was blind, or because of some uncertainty about the diagnosis, or because a few clinicians in recent years have not been convinced that the traditional ophthalmological practice of treating all melanomas is proper, there is now a growing body of evidence that most of these tumours show little or no growth over extended periods of careful clinical evaluation, and that only very rarely does one hear of cases in which the tumour has metastasised during such periods of clinical study (Fuchs, cited by Parsons, 1905; Zimmerman and Adler, 1957; Duke-Elder and Perkins, 1966; Raivio, 1977; Ruiz, 1977). Raivio (1977), who recently reported on a comprehensive analysis based on 378 uveal melanoma cases in Finland, found that there were 32 patients whose tumour-containing eyes had been observed for some time prior to enucleation. Although evidence of growth had been found in each instance, no spread had been observed, and the survival rates after enucleation for these patients were no different from those of other patients whose eyes had been enucleated promptly after discovery of the tumours. In addition, there were 5 patients who had refused enucleation. One died of unrelated causes after 1 year. The other 4 patients eventually died of metastatic disease, but of great interest is the fact that in none of these cases did the tumour prove lethal in less than 2 years, the deaths having occurred after $2,3,14$, and 15 years.

Our follow-up data (Fig. 1) and those of other investigators (Benjamin et al., 1948; Chisholm, 1953; Westerveld-Brandon and Zeeman, 1957; Jensen, 1963) show that there is a significant mortality that becomes evident after enucleation. If the evidence that there is little mortality before enucleation is accepted, this suggests that enucleation adversely affects the course of many uveal melanomas. One could design a study to test this hypothesis, but it would require a large series of unselected, clinically diagnosed melanomas left untreated and followed up for long periods. In contemplating the possible results of such a study, we have constructed several possible survival curves (Fig. 2).

The solid line (curve $E-P$ ) in Fig. 2 represents survival after enucleation of the 2652 patients in the study of Paul and co-workers (1962). The curves shown as broken lines are all hypothetical. The curve $A-E$ with a shallow slope equivalent to a death rate of $1 \%$ per year represents our estimate of the low mortality from unrecognised and untreated uveal melanomas. One might assume that the mortality rate of uveal melanomas would not change once the tumours have been recognised, even though they are left untreated; thus the continuation of line $A-E$ as $E-A^{\prime}$ with the same slope represents that possibility. On the other hand it is possible that after a variable period of time one or more biological changes, for 


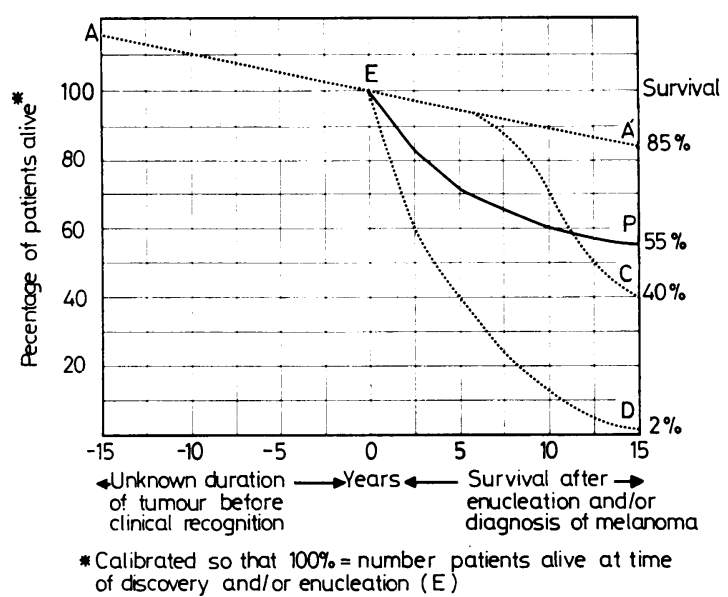

Fig. 2 Actual and hypothetical survival curves for patients with malignant melanoma of the choroid and ciliary body. $\mathrm{E}$ is that point in the course of the tumour when enucleation was performed in those cases represented by actual survival curve E-P, and when the tumour was discovered in the hypothetical non-treated cases represented by broken lines. E-P is the actuarial survival curve for the 2652 cases treated by enucleation (based on data published by Paul et al. (1962). A-E is the hypothetical survival curve of patients with undiagnosed uveal melanomas based on an estimated tumour death rate of $1 \%$ per year. $\mathrm{E}-\mathrm{A}^{\prime}$ is the continuation of $\mathrm{A}-\mathrm{E}$ with the same slope based on the assumption that if the tumours were left untreated after recognition, the death rate would not change. $\mathrm{E}-\mathrm{C}$ is the hypothetical survival curve of untreated patients based on the assumption that eventually a change would take place that would result in an increased death rate. E-D is the hypothetical survival curve of untreated patients based on the assumption that if enucleation is not performed when the tumour is recognised, most patients would soon die of metastatic disease. AFIP Neg. 78-206

example, mutation to a more malignant cell type, depression of immunological defence mechanisms, etc., might take place that would accelerate the mortality rate. That possibility is represented by curve $E-C$. The third possibility is represented by curve $E-D$, which shows a steep downward slope beginning soon after the time of discovery of the tumour.

Of these various hypothetical possibilities we believe the third represented by curve $E-D$ is the least plausible. If this hypothesis were correct, then the discovery of the presence of a melanoma would be associated with a sudden tendency toward dissemination and a lethal outcome. This is to say that the same factors that lead to recognition of a melanoma also predispose the tumour to metastasise and to have a higher mortality rate. The flaw in the logic of this argument is that melanomas are not recognised at a specific time during their development. Some are discovered in the course of a routine examination of an asymptomatic patient while others come to the attention of an ophthalmologist after a delay of years and only after various complications have developed. If the sudden increase in risk of metastasis were associated with some clinical factor leading to enucleation, then cases in which there is a delay after the onset of symptoms should have an appreciable preoperative mortality. A high preoperative mortality among patients in whom enucleation is delayed or not performed has neither been our experience nor has it been reported by others, and an increasing body of evidence based on totally untreated patients and on others who were followed up for 1 or more years before enucleation can be marshalled in support of this conviction.

Isolated cases can be cited in support of the possiblities represented by curves $E-A^{\prime}$ and $E-C$. Having discarded the possibility that curve $E-D$ would represent survival of patients with untreated uveal melanomas, let us now compare the actual survival curve $E-P$, representing results achieved with enucleation, with curves $E-A^{\prime}$ and $E-C$. It is obvious that the survival of untreated patients represented by curve $E-A^{\prime}$ is far better than that of the treated patients in the series reported by Paul et al. (1962). It is also evident that for the first 11 years the hypothetical survival of untreated patients represented in curve $E-C$ is also better than that of the treated patients in curve $E-P$.

From the data of Paul et al. (1962) used to construct the survival curve $E-P$ we have calculated the changing annual mortality rate following enucleation to construct the graph shown in Fig. 3. This reveals more dramatically the rapid rise in mortality that follows enucleation, reaching a peak during the second postenucleation year. Then during the ensuing several years the tumour death rate falls, reaching a low, apparently constant level about 6 to 7 years after enucleation. After having made these observations we discovered that Benjamin and co-workers (1948) in the follow-up study of a large series of British cases published a graph revealing a remarkably similar pattern. In a discussion of metastatic disease from uveal melanomas Jensen (1963) presented a graph which again reflects this striking pattern of a rapidly rising incidence after enucleation and reaching a peak 2 to 3 years after enucleation.

Again one cannot avoid making the comparison with cases that have been followed up without enucleation and in which there may be clinical evidence of progressive growth accompanied by such complications as intraocular haemorrhage, secondary glaucoma, and/or extraocular extension, 


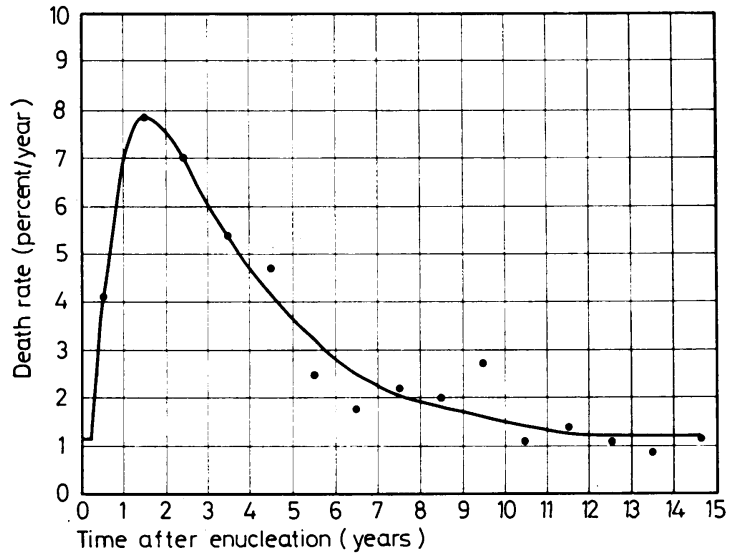

Fig. 3 Relationship of annual death rate to time after enucleation. After a lag period of about 3 months the death rate rises abruptly, reaching a peak of about $8 \%$ per year during the second year after enucleation. It then falls off over a period of several years, reaching the estimated pre-enucleation rate of about $1 \%$ per year. AFIP Neg. 78-205

yet in such cases the patient rarely shows evidence of metastatic disease during the first several years of observation. One comes up with the distinct impression that the process of surgically removing the tumour-containing eye may promote dissemination of tumour cells.

Fraunfelder and co-workers (1977) have for several years suspected that this might be the case and they have designed experiments to test the hypothesis (Burns et al., 1962; Fraunfelder and Boozman, 1974). They showed that, while a quick atraumatic method of enucleation of the hamster eye tended to prevent dissemination of tumour cells, the conventional surgical technique was less effective. However, neither method of enucleation was curative except in a few animals. More recently they have shown that the conventional techniques for enucleation are accompanied by great fluctuations in intraocular pressure, which could, along with the traumatic effects of direct handling of the tissues, be responsible for a considerable increase in intravasation of tumour cells (Fraunfelder et al., 1977). Such a release of melanoma cells into the circulation during enucleation might well overwhelm whatever beneficial immunological defence mechanisms the patient may have mobilised prior to enucleation.

From these assumptions we have formulated a mathematical model to describe the mortality rate observed with uveal melanomas treated by enucleation. Features and assumptions incorporated into the model are:

(1) The death rate is equal to the metastatic rate, i.e., the rate at which tumour emboli become established as metastatic foci and start growing in distant sites. But obviously there is a lag period between time of metastasis and the time of death, as it takes time for established metastatic foci to grow and kill the patient.

(2) The shower of emboli released at enucleation causes the rate of metastasis to increase exponentially up to a maximum level.

(3) Counteracting the shower of emboli and the metastatic foci are host defences which attempt to destroy the released tumour cells at an exponential rate.

(4) There is a constant mortality rate that is independent of enucleation. The model is:

Death rate $=M\left(1-\mathrm{e}^{-\mathrm{k}_{\mathrm{m}}(\mathrm{t}-\mathrm{L})}\right) \mathrm{e}^{-\mathrm{k}_{\mathrm{d}}(\mathrm{t}-\mathrm{L})}+\mathrm{C}$ where,

$\mathbf{M}=$ Maximum increase in death rate attributable to release of tumour cells at enucleation.

$\mathrm{k}_{\mathrm{m}}=$ Rate of metastasis of tumour.

$k_{\mathbf{d}}=$ Rate of destruction of tumour cells released into circulation and of metastases.

$\mathrm{t}=$ Length of time after enucleation.

$\mathrm{L}$ = Lag-time from establishment of metastatic foci to death from tumour.

$\mathrm{C}=$ Death rate independent of enucleation.

The curve shown in Fig. 3 is the least-square fit to the observed death rate using this model. The calculated values for the parameters are: $M=17.8 \% /$ year $; \mathrm{k}_{\mathrm{m}}=0.80 /$ year $; \mathrm{k}_{\mathrm{d}}=0.41 /$ year; $\mathrm{L}=0.24$ years; $\mathrm{C}=1 \cdot 14 \% /$ year.

If the medical concepts underlying this model represent a reasonable description of events following enucleation, then emboli released at the time of enucleation could account for two-thirds of the $45 \%$ mortality observed at 15 years. This estimate is based on the approximately $1 \%$ constant rate over 15 years $(15 \%)$, which is then subtracted from the final $45 \%$ mortality to give $30 \%(45 \%-15 \%=$ $30 \%$ ). Thus two-thirds of the overall mortality $(30 \% / 45 \%)$ is attributable to enucleation. By the same reasoning these data suggest that a method of enucleation that would not disseminate cells could reduce the 15 -year mortality to about $15 \%$.

These calculations have been based on survival data derived from a large unselected series of cases treated by enucleation. The question might arise as to how they relate to our previous studies of prognostic factors that have shown that patients with large melanomas have a worse prognosis than patients with small melanomas, and that patients with tumours containing epithelioid cells have a worse prognosis than patients with pure spindle cell tumours (Flocks et al., 1955; McLean et al., 1977). All such studies have also been retrospective and based entirely on cases treated by enucleation. Thus the basic principles would still apply, but the adverse effects of enucleation would be greater if one 
restricted the analysis to large tumours or to tumours containing epithelioid cells. On the other hand one would encounter the least chance of disseminating tumour cells if one selected only small spindle cell tumours for enucleation. This provides another explanation for the excellent follow-up results obtained in the treatment of melanomas of the iris (Ashton, 1964; Zimmerman, 1971-72).

The suggestion of Fraunfelder et al. (1977) that we need some sort of 'no-touch technique' for enucleation must be heeded. A first step, however, is to develop a new philosophy regarding all sorts of manipulation of the eye containing a melanoma. This would include curtailment of repeated examinations by the entire resident staff and miscellaneous visiting ophthalmologists who might want to use such aids as vigorous scleral depression and transillumination during their examinations. It would also require a change in the policy of many teaching institutions that assign to first-year residents, who have the least surgical expertise, the responsibility for performing enucleations.

Many recent articles have indicated that because of the lack of controlled studies we do not have proof of the benefits derived from enucleation in the treatment of uveal melanomas, but only rarely has it been suggested that the surgeon may actually hasten the dissemination of tumour while performing an enucleation (Westerveld-Brandon and Zeeman, 1957; Zimmerman and McLean, 1975; Fraunfelder et al., 1977). According to the Hippocratic doctrine physicians should at least do no harm (Blomquist, 1977). It is time we tried to learn exactly what we are doing for (or against) the patient when we recommend enucleation for uveal melanomas.

The opinions or assertions contained herein are the private views of the authors and are not to be construed as official or as reflecting the views of the Department of the Army or the Department of Defense.

This study was supported in part by research grant EY-01386 from the National Institutes of Health, DHEW.

\section{References}

Anderson, B., and O'Neill, J. (1957). Malignant melanoma of the uvea; observations based on growth and behavior, enucleation refused or delayed. Archives of Ophthalmology, 58, 337-347.

Ashton, N. (1964). Primary tumours of the iris. British Journal of Ophthalmology, 48, 650-668.

Benjamin, B., Cumings, J. N., Goldsmith, A. J. B., and Sorsby, A. (1948). Prognosis in uveal melanoma. British Journal of Ophthalmology, 32, 729-747.
Blomquist, C. D. D. (1977). From the oath of Hippocrates to the declaration of Hawaii. Ethics in Science and Medicine, 4, 139.

Burns, R. P., Fraunfelder, F. T., and Klass, A. M. (1962). A laboratory evaluation of enucleation in treatment of intraocular malignant melanoma. Archives of Ophthalmology, 67, 490-500.

Char, D. H., and Hogan, M. J. (1977). Management of small elevated pigmented choroidal lesions. British Journal of Ophthalmology, 61, 54-58.

Chisholm, J. F. (1953). A long term follow-up on malignant melanomas of the choroid based on the Terry and Johns series. American Journal of Ophthalmology, 36, 61-73.

Curtin, V. T. (1977). Malignant melanoma management. In Controversy in Ophthalmology, p. 635. Edited by R. J. Brockhurst, S. A. Boruchoff, B. T. Hutchinson, and S. Lessell. Saunders: Philadelphia.

Duke-Elder, S., and Perkins, E. S. (1966). Diseases of the uveal tract. In Duke-Elder, S. System of Ophthalmology, p. 871. Henry Kimpton: London.

Dunphy, E. B. (1977). A conservative approach to the malignant melanoma problem. In Controversy in Ophthalmology: p. 611. Edited by R. J. Brockhurst, S. A. Boruchoff: B. T. Hutchinson, and S. Lessell. W. B. Saunders: Philadelphia.

Flocks, M., Gerende, J. H., and Zimmerman, L. E. (1955). The size and shape of malignant melanomas of the choroid and ciliary body in relation to the prognosis and histologic characteristics. A statistical study of 210 tumors. Transactions of the American Academy of Ophthalmology and Otolaryngology, 59, 740-758.

Fraunfelder, F. T., and Boozman, F. W., (1974). 'No-touch techniques' for management of ocular malignant melanomas. Association for Research in Vision and Ophthalmology, Annual Meeting, 2 April, Sarasota, Florida.

Fraunfelder, F. T., Boozman, F. W., Wilson, R. S., and Thomas, A. H. (1977). No-touch technique for intraocular malignant melanomas. Archives of Ophthalmology, 95, 1616-1620.

Gass, J. D. M. (1977). Problems in the differential diagnosis of choroidal nevi and malignant melanomas. American Journal of Ophthalmology, 83, 299-323.

Hagler, W. S., Jarrett W. H., and Killian, J. H. (1977). The use of P-32 test in the management of malignant melanoma of the choroid: a five-year follow-up study. Transactions of the American Academy of Ophthalmology and Otolaryngology, 83, 49-60.

Hogan, M. J. (1964). Clinical aspects, management, and prognosis of melanomas of the uvea and optic nerve. In Ocular and Adnexal Tumors, New and Controversial Aspects, p. 257. Edited by M. Boniuk. Mosby: St. Louis.

Jensen, O. A. (1963). Malignant melanomas of the uvea in Denmark, 1943-1952; a clinical, histopathological, and prognostic study. Acta Ophthalmologica, Suppl. 75.

Koenig, I. J. (1954). Malignant melanoma of the iris and ciliary body of a one-eyed patient. Archives of Ophthal. mology, 51, 656.

McLean, I. W., Foster, W. D., and Zimmerman, L. E. (1977). Prognostic factors in small malignant melanomas of choroid and ciliary body. Archives of Ophthalmology, 95, 48-58.

Parsons, J. H. (1905). The Pathology of the Eye, Vol. 2, Ch. 9, p. 500. Putnam: New York.

Paul, E. F., Parnell, B. C., and Franker, M. (1962). Prognosis of malignant melanomas of the choroid and ciliary body. International Ophthalmology Clinics, 2, 387-402.

Raivio, I. (1977). Uveal melanoma in Finland. An epidemiological, clinical, histological and prognostic study. Acta Ophthalmologica, Suppl. 133.

Ruiz, R. S. (1977). Early treatment in malignant melanoma 
of the choroid. In Controversy in Ophthalmology, p. 604. Edited by R. J. Brockhurst, S. A. Boruchoff, B. T. Hutchinson, and S. Lessell. Saunders: Philadelphia.

Westerveld-Brandon, E. R., and Zeeman, W. P. C. (1957). The prognosis of melanoblastomata of the choroid. Ophthalmologica, 134, 20-29.

Zimmerman, L. E. (1967). Changing concepts concerning the malignancy of ocular tumors. Archives of Ophthalmology, 78, 166-173.

Zimmerman, L. E. (1971-72). Histopathologic considerations in the management of tumors of the iris and ciliary body. Anales del Instituto Barraquer, 10, 27-57.

Zimmerman, L. E., and Adler, F. H. (1957). Clinical Pathologic Conference. American Journal of Ophthalmology, 43, 223-231.

Zimmerman, L. E., and McLean, I. W. (1975). Changing concepts of the prognosis and management of small malignant melanomas of the choroid. Transactions of the Ophthalmological Societies of the United Kingdom, 95, 487-494. 